\title{
Anomalous X\#Ray Pulsars and Soft Gamma\#Ray Repeaters: Spectral Fits and the Magnetar Model
}

\section{Citation}

Perna, Rosalba, Jeremy S. Heyl, Lars E. Hernquist, Adrienne M. Juett, and Deepto Chakrabarty. 2001. "Anomalous X\#Ray Pulsars and Soft Gamma\#Ray Repeaters: Spectral Fits and the Magnetar Model." The Astrophysical Journal 557 (1): 18-23. https://doi.org/10.1086/321569.

\section{Permanent link}

http://nrs.harvard.edu/urn-3:HUL.InstRepos:41381698

\section{Terms of Use}

This article was downloaded from Harvard University's DASH repository, and is made available under the terms and conditions applicable to Other Posted Material, as set forth at http:// nrs.harvard.edu/urn-3:HUL.InstRepos:dash.current.terms-of-use\#LAA

\section{Share Your Story}

The Harvard community has made this article openly available.

Please share how this access benefits you. Submit a story.

Accessibility 


\title{
Anomalous X-ray Pulsars and Soft $\gamma$-ray Repeaters: Spectral Fits and the Magnetar Model
}

\author{
Rosalba Perna, ${ }^{1}$ Jeremy S. Heyl, ${ }^{2}$ and Lars E. Hernquist \\ Harvard-Smithsonian Center for Astrophysics, 60 Garden Street, Cambridge, MA 02138; \\ rperna@cfa.harvard.edu, jheyl@cfa.harvard.edu, lhernqui@kona.harvard.edu \\ and \\ Adrienne M. Juett and Deepto Chakrabarty \\ Department of Physics and Center for Space Research, Massachusetts Institute of Technology, Cambridge, \\ MA 02139; ajuett@space.mit.edu,deepto@space.mit.edu
}

\begin{abstract}
The energy source powering the X-ray emission from anomalous X-ray pulsars (AXPs) and soft $\gamma$-ray repeaters (SGRs) is still uncertain. In one scenario, the presence of an ultramagnetized neutron star, or "magnetar", with $B \simeq 10^{14}-10^{15} \mathrm{G}$ is invoked. To investigate this hypothesis, we have analyzed archival $A S C A$ data for several known AXPs and SGRs, and fitted them with a model in which all or part of the X-ray flux originates as thermal emission from a magnetar. Our magnetar spectral model includes the effects of the anisotropy of the heat flow through an ultramagnetized neutron star envelope, reprocessing by a light element atmosphere, and general relativistic corrections to the observed spectrum. We obtain good fits to the data with radii for the emitting areas which are generally consistent with those expected for neutron stars, in contrast to blackbody (BB) fits, which imply much smaller radii. Furthermore, the inclusion of atmospheric effects results in inferred temperatures which are lower than those implied by BB fits, but however still too high to be accounted by thermal cooling alone. An extra source of heating (possibly due to magnetic field decay) is needed. Despite the harder tail in the spectrum produced by reprocessing of the outgoing flux through the atmosphere, spectral fits still require a considerable fraction of the flux to be in a power-law component.
\end{abstract}

Subject headings: stars: neutron $-X$-rays: stars

\footnotetext{
${ }^{1}$ Harvard Junior Fellow

${ }^{2}$ Chandra Fellow
} 


\section{Introduction}

The roughly half dozen so-called anomalous X-ray pulsars (AXPs; Mereghetti \& Stella 1995; van Paradijs, Taam, \& van den Heuvel 1995) have recently emerged as a distinct class of objects. They are slow $(P \sim 5-10 \mathrm{~s})$ rotators with no evidence of Doppler shifts from binary motion, and several are associated with supernova remnants, suggesting that they are young objects. Owing to their long periods, their rotational energy loss is far too low to power their observed X-ray luminosities. Models to account for the properties of these sources fall into two broad categories. In the first class of models, the X-ray emission is powered by accretion, which could result from a binary companion of very low mass (Mereghetti \& Stella 1995), the debris of a disrupted massive companion (van Paradijs, Taam \& van den Heuvel 1995; Ghosh, Angelini \& White 1997), or material falling back after a supernova explosion (Corbet et al. 1995; Chatterjee, Hernquist \& Narayan 2000; Alpar 1999, 2000; Marsden et al. 2001). In the second class of models, accretion is not involved; instead, the AXPs are hypothesized to be either ultramagnetized neutron stars (NSs; Thompson \& Duncan 1996) or remnants of Thorne-Żytkow objects (van Paradijs et al. 1995). In the former case, the X-ray luminosity could be powered either by magnetic field decay (Thompson \& Duncan 1996; Heyl \& Kulkarni 1998) or by residual thermal energy (Heyl \& Hernquist 1997a,b).

If the AXPs are indeed ultramagnetized NSs (or "magnetars"; Duncan \& Thompson 1992, 1995), then they may be related to another class of objects, the soft $\gamma$-ray repeaters (SGRs; e.g. Kouveliotou et al. 1999). In their quiescent X-ray emission, the SGRs have slow pulsations similar to those seen in the AXPs. In addition, the SGRs sometimes show strong hard X-ray/soft gammaray bursts, which can be distinguished from the classical $\gamma$-ray bursts (GRBs) by their recurrence and their spectra, which are generally much softer than those of the GRBs. According to Thompson \& Duncan (1996), these bursts could be the result of sudden releases of energy resulting from rearrangement of the magnetic fields in the crusts of highly magnetized NSs.

In the magnetar model for the AXPs and SGRs, whether the X-ray luminosity is powered by cooling or magnetic field decay or by a combination of both, the thermally emitting area must be consistent with a neutron star surface. However, blackbody fits to the spectra of these objects require emitting areas that are only a small fraction of the total surface. This discrepancy may arise at least partially because the thermal emission of NSs most likely does not have a true blackbody spectrum. Atmospheric effects are known to distort NS spectra. Fallback after the supernova explosion, and/or accretion from the interstellar medium, will likely cover the surface of the star with light elements. (Note that to cover the surface to an X-ray optical depth of unity requires only $\sim 10^{14} \mathrm{~g}-10^{-19} M_{\odot}$ of material.) Moreover, this material is likely to suffer significant fractionization on short timescales (Alcock \& Illarionov 1980; Romani 1987). Owing to the enormous surface gravity of the star, the heavier elements will settle out in of order 1-100 sec, leaving an atmosphere made of light elements. Besides atmospheric effects, the emergent NS spectrum is also dependent on the underlying temperature distribution on the stellar surface (in blackbody fits this is assumed incorrectly to be uniform), and on the general relativistic effect of light deflection owing to the large surface gravity. A spectral analysis that takes into account all 
these effects is the purpose of this work.

More specifically, in this Letter, we analyze archival $A S C A$ data for some of the known AXPs and SGRs, and fit them with a model in which the X-ray flux is produced by thermal emission from a highly magnetized NS with an atmosphere made of light elements. We take into account the anisotropy in the flow of heat through the envelope of the NS owing to the intense magnetic field, as well as general relativistic corrections to the observed spectrum. Our fits are consistent with emission from the entire surface of a neutron star, supporting the interpretation of AXPs and SGRs as magnetars.

\section{The X-Ray Spectrum of a Cooling Magnetar}

We consider a highly magnetized neutron star cooling through an accreted envelope. Heyl \& Hernquist $(1998 \mathrm{~b}, 2000)$ showed that, if $B_{p} \gtrsim 10^{12} \mathrm{G}$, the flux transmitted through the envelope can be well approximated by $F \propto \cos ^{2} \psi$, where $\psi$ is the angle between the local radial direction and the magnetic field. For a dipolar field,

$$
\cos ^{2} \psi=4 \cos ^{2} \theta_{p} /\left(3 \cos ^{2} \theta_{p}+1\right)
$$

(Greenstein \& Hartke 1983), where $\theta_{p}$ is the angle between the radial direction at position $(\theta, \phi)$ on the surface of the star, and the magnetic pole. In spherical coordinates, it is given by

$$
\cos \theta_{p}=\cos \theta \cos \alpha+\sin \theta \sin \alpha \cos \phi,
$$

where $\alpha$ is the angle that the magnetic pole makes with the line of sight. For this study, we consider an orthogonal rotator. 3 The angle $\alpha$ is then coincident with the phase angle $\Omega t$, where $\Omega$ is the angular velocity of the star. For the local emission from the NS surface, $n(E, T)$, we assume a blackbody spectrum modified by the presence of an atmosphere made of light elements, for which we adopt the semianalytical model of Heyl \& Hernquist (1998a), but with the inclusion of limb darkening. This is parameterized by an angle dependence of the intensity $\propto \cos ^{\beta} \delta$. The dependence on $\beta$ is explored in the fits.

Let $R$ be the radius of the NS star, $M$ its mass (for which we adopt $M=1.4 M_{\odot}$ ), $R_{s}=2 G M / c^{2}$ its Schwarzschild radius, and define $e^{-\Lambda_{s}} \equiv \sqrt{1-R / R_{s}}$. If $D$ is the distance from the star to the observer, the phase-averaged flux measured by an observer at infinity (without including the

\footnotetext{
${ }^{3}$ We find that spectral fits are insensitive to this choice when considering the average flux over the rotational period of the star.

${ }^{4}$ This model yields spectral intensities very close to those of Pavlov et al. (1994), who computed detailed atmospheres for magnetic field strengths on the order of $10^{12}-10^{13} \mathrm{G}$. Preliminary results (D. Lloyd et al. in preparation) suggest furthermore that realistic spectra are similar to ours even for much stronger fields, $B \sim 10^{14}-10^{15} \mathrm{G}$.
} 
reduction due to photoelectric absorption by intervening material) is given by

$$
f(E)=\frac{\pi R_{\infty}^{2} \sigma T_{p, \infty}^{4}}{4 \pi D^{2}} \frac{1}{k T_{p, \infty}} \int_{0}^{2 \pi} \frac{d \alpha}{2 \pi} \int_{0}^{1} 2 x d x \int_{0}^{2 \pi} \frac{d \phi}{2 \pi} I_{0}(\theta, \phi) n\left[E e^{-\Lambda_{s}} ; T_{s}(\theta, \phi)\right],
$$

in units of photons $\mathrm{cm}^{-2} \mathrm{~s}^{-1} \mathrm{keV}^{-1}$. Here $x=\sin \delta$ ( $\delta$ being the angle between the normal to the NS surface and the direction of the photon trajectory), $R_{\infty} \equiv R e^{\Lambda_{s}}$, and $T_{p, \infty} \equiv T_{p} e^{-\Lambda_{s}}$, where

$T_{p}$ is the temperature at the pole. The general relativistic effects of light deflection are taken into account through the ray-tracing function (Page 1995)

$$
\theta(\delta)=\int_{0}^{R_{s} / 2 R} x d u / \sqrt{\left(1-\frac{R_{s}}{R}\right)\left(\frac{R_{s}}{2 R}\right)^{2}-(1-2 u) u^{2} x^{2}},
$$

where $u=R_{s} / 2 r$, with $r$ being the radial coordinate. A photon emitted at an angle $\delta$ with respect to the normal to the surface comes from a colatitude $\theta(\delta)$ on the star. The total flux at each point $(\theta, \phi)$ of the NS surface is given by

$$
I_{0}(\theta, \phi)=\frac{4 \cos ^{2} \theta_{p}}{3 \cos ^{2} \theta_{p}+1}\left(0.75 \cos ^{2} \theta_{p}+0.25\right)^{0.2} .
$$

The first term in the right hand side is appropriate for a dipole, and we have then assumed a further dependence of the flux on $B^{0.4}$, as in Heyl \& Hernquist (1998b). Finally, the local temperature on the stellar surface is determined by

$$
T_{s}(\theta, \phi)=T_{p}\left[I_{0}(\theta, \phi)\right]^{1 / 4}
$$

\section{Observations and Analysis}

We analyzed archival observations of several AXPs and SGRs made by the Advanced Satellite for Cosmology and Astrophysics (ASCA; Tanaka, Inoue, \& Holt 1994), obtained from the High Energy Astrophysics Archival Research Center (HEASARC) at NASA Goddard Space Flight Center. ASCA was launched in 1993 and continued to make observations through 2000. It carried four identical grazing incidence X-ray telescopes capable of imaging X-rays in the $0.5-10 \mathrm{keV}$ range with a $24 \operatorname{arcmin}$ (FWHM) field of view, a $\sim 1$ arcmin point spread function, and a total effective area of $1300 \mathrm{~cm}^{2}$ at $1 \mathrm{keV}$. Each telescope had one dedicated focal plane instrument, and all four instruments simultaneously recorded data for each observation. The two CCD cameras (SIS0 and SIS1) had superior energy resolution $(E / \Delta E \sim 20-50)$ and were sensitive down to about $0.5 \mathrm{keV}$. The two gas scintillation imaging proportional counters (GIS2 and GIS3) had more modest energy resolution $(E / \Delta E \sim 10)$ and little sensitivity below $1 \mathrm{keV}$, but their effective area was comparable to the CCDs around $2 \mathrm{keV}$ and higher above $\sim 4 \mathrm{keV}$. Thus, the GIS data were somewhat better suited for studying the X-ray continuum spectrum of absorbed sources like the AXPs and SGRs. However, we initially examined data from all four instruments in our analysis. 
A summary of the $A S C A$ observations that we analyzed is given in Table 1. For simplicity, we confined ourselves to those sources where there is no contaminating emission from a surrounding supernova remnant to consider; this excludes the AXPs 1E 2259+586 and 1E 1841-045. We used the standard screened events files provided through HEASARC by the ASCA Guest Observer Facility (GOF). From these files, spectra were extracted for the point sources. The extraction radii were 6 arcmin and 4arcmin for GIS and SIS respectively. Background spectra were also extracted from the event files from the area outside a 8 arcmin radius from the point source and any other bright sources present in the observation. We fitted the X-ray spectra for all the observations in Table 1 using the XSPEC spectral analysis package (Arnaud 1996). In each case, we fit the analytic magnetar model described in Equation (3). For comparison, we also fit an ideal blackbody model (BB), assuming a uniform temperature distribution over the emitting area. The free parameters of the magnetar model are the pole temperature $T_{p}$ and the radius of the star $R$. (For a nonrelativistic star, the radius affects only the overall normalization of the spectrum; however, when general relativistic effects are taken into account, the radius also modifies the shape of the spectrum through the dependence of the function $\theta(x)$ in Equation (3) on $R$.)

For both the magnetar and the BB models, we included the multiplicative effect of interstellar photoelectric absorption (Morrison \& McCammon 1983). We also allowed for the possibility of an absorbed power law (PL) component, using the same absorption column as for the thermal component. However, for each object, a fit using only the absorbed thermal component alone was also attempted. A spectrum produced by an atmosphere indeed has a harder tail compared to simple blackbody emission at the same effective temperature. Therefore, even if the blackbody fit always required an extra power law component, this did not necessarily have to be the case when processing by an atmosphere was included in the computation of the spectrum.

For each observation, we began by fitting the data for each instrument individually, in order to evaluate the data quality separately. If the data from one or more of the instruments had poor statistics (usually in the case of the SIS data) or was otherwise problematic, it was discarded. The remaining data were then fit jointly to maximize our continuum sensitivity, with the overall normalization tied to that of the GIS2 detector (which generally gave the best independent fits). In the case of the SGR 1627-41 and SGR 1806-20, we found that there were too few soft counts to allow a meaningful constraint on the fit parameters for the thermal components. We do not consider these sources further in this paper.

The results of our fits are summarized in Table 2. For each source, we show both the absorbed $\mathrm{BB}+\mathrm{PL}$ fits and the absorbed magnetar+PL fits. In the case of AX J1845-0258, however, the statistics were too poor to constrain both components separately. For this object, the thermal and non-thermal components in Table 2 represent alternate fits to the same data. In a few cases (i.e. when the fit was acceptable), we also show an absorbed magnetar-PL fit with the column density held fixed at $N_{\mathrm{H}}=1 \times 10^{22} \mathrm{~cm}^{-2}$. For all our fits, we also give the fraction of the flux that is in the power law component, computed in terms of the unabsorbed photon flux in the $0.7-10 \mathrm{keV}$ band. A comparison between the temperatures and radii obtained in the BB fits and in the fits with the 
atmospheres is shown in Figure 1 (except for the object RXS J1708-40, whose distance is still largely uncertain, and for which a separate discussion will be made in $\S 4$ ). The two dashed lines mark the region of NS radii which are allowed by currently available models for the NS equation of state.

\section{Discussion}

We have analyzed archival $A S C A$ data for several known AXPs and SGRs and fitted their spectra with a model in which the X-ray emission consists of thermal radiation from a highly magnetized neutron star, as well as a power-law component at high energies. For the thermal contribution, we have included distortions in the spectra due to the presence of an atmosphere of light elements, accounted for the anisotropic flow of heat through the envelope due to the intense magnetic field, and included general relativistic corrections to the observed flux. We find that the thermal emitting areas implied by our model are always larger than those derived by spectral fits that use a blackbody spectrum, and are generally consistent with those expected for neutron stars. All the fits were made using a model with a moderate beaming, i.e. $\beta \sim 0-1$. We have considered other radiation patterns and find that a more intense beaming results in slightly larger inferred areas and smaller temperatures, but the fits are equally acceptable. Therefore, spectral fits alone made with the phase averaged spectrum are not able to constrain the degree and type of beaming, especially given the uncertainties in the other parameters.

In most of the cases we found that, despite the fact that processing by an atmosphere leads to lower temperatures than those implied by the BB fits, our inferred temperatures are still too high to be accounted for by thermal cooling alone. However, if the magnetic field is sufficiently strong and decaying, the energy from its decay may augment the thermal emission from the surface (Heyl \& Kulkarni 1998). The inferred surface temperatures of all of the objects with well constrained fits are higher than would be expected for a neutron star cooling through an iron envelope (Heyl \& Hernquist 2000); the results for the AXP 4U 0142+61, RXS J1708-40 and SGR 1900+14 are marginally consistent with a neutron star cooling through a highly magnetized iron envelope with a substantial contribution to the flux from the decay of the magnetic field (Heyl \& Kulkarni 1998). If the energy released by the magnetic field is deposited at high densities, magnetic field decay alone cannot explain the high effective temperatures of the AXPs 1E 1048.1-5937 and 1E 1845-0245. Thermal emission through a light element envelope (Heyl \& Hernquist 1997b, Potekhin, Chabrier \& Yakovlev 1997), however, can account for these sources.

Atmospheric effects play a crucial role in determining the emitted spectrum (Romani 1987; Pavlov et al. 1994; Zampieri et al. 1995; Rajagopal \& Romani 1996; Zavlin, Pavlov \& Shibanov 1996). For the effective temperatures of these stars (i.e. $\sim$ a few $\times 10^{6} \mathrm{~K}$ ), the spectra from hydrogen atmospheres depend only weakly on the strength of the magnetic field (see e.g. Fig. 5 in Rajagopal, Romani \& Miller 1997). However, the composition of the atmosphere may dramatically affect the emitted spectrum (see, e.g. references above). If the atmosphere were made of heavy 
elements such as iron, the emitted spectrum would be much closer to a blackbodyf (Rajagopal et al. 1997).

Note that, given the mild dependence of the spectrum on the strength of the magnetic field for $B \gtrsim 10^{12} \mathrm{G}$, the model we have developed could similarly be used to model the thermal emission of neutron stars with magnetic fields $\sim 10^{12}-10^{13} \mathrm{G}$ and light element atmospheres. However, in the case of the AXPs, the additional heat (with respect to the predictions of standard cooling scenarios) would be difficult to explain without the contribution from magnetic field decay. A hypothetical contribution from accretion would most likely result from matter channeled onto the magnetic poles of the star by the presence of the magnetic field lines. This would yield hot polar caps and the total spectrum would show two thermal components, one at lower energy being consistent with the total area of the star (due to thermal cooling) and another at higher temperature but coming from a small fraction of the star (due to the heated polar caps). Here we find that spectral fitting requires only one thermal component, and therefore this scenario does not seem to be favored. However, it is also true that the high column density to these sources would make a low-temperature thermal component difficult to detect.

The distances to the sources are still rather uncertain. As Figure 1 shows, relatively small variations with respect to the assumed values would not affect any of the conclusions of this work. The value of the inferred radius, $R$, roughly scales with the distance $D$. If any of the distances turned out to be much smaller than the assumed value, so that the radii were consequently much smaller than the minimum allowed value by the NS equation of state, then an accretion model where the X-ray emission is produced by a hot spot would be favored with respect to the magnetar model, where it is produced by the whole surface of the star. On the other hand, if any of the distances turned out to be much larger than the assumed value, so that the corresponding BB radius were already consistent with the emission from the entire surface, then a light element atmosphere would not be appropriate, as it would yield too large radii, while an atmosphere made of heavy elements such as iron would be a viable option, because it yields spectra very close to blackbody, as discussed above. In such a case, it would be difficult to interpret the X-ray emission as being powered by accretion unless the magnetic field of the objects were very small, so that accretion could proceed in a quasi-spherical fashion rather than being channeled through the poles .

Among all the objects that we considered, RXS J1708-40 is the one which has the largest uncertainty in its distance. It lies in the Galactic plane, and, along this direction, spiral arms are located at a distance of $\sim 1, \sim 3$, and $\sim 4.5 \mathrm{kpc}$ (Taylor \& Cordes 1993). The large column density to the source inferred from the X-ray spectra suggests a likely distance in the 5 - $10 \mathrm{kpc}$ range (Israel

\footnotetext{
${ }^{5}$ Note that the accretion model predicts that the opacity should be dominated by heavy metals in SGRs and AXPs, because the settling time is dominated by the rate of metal deposition for the accretion rates implyed by the X-ray emission (Brown, Bildsten \& Rutledge 1998).

${ }^{6}$ Note that in models where accretion derives from fallback disks (e.g. Chatterjee et al. 2000), a magnetic field of the order of $10^{12}-10^{13} \mathrm{G}$ is needed.
} 
et al. 1999). However, a smaller distance cannot be ruled out. Our results suggest that this object, if it were a magnetar with a light element atmosphere, would most likely reside in the middle spiral arm. However, a magnetar with an atmosphere of heavy elements would be consistent with the largest estimates of the distance.

Besides the distance to the sources, the main uncertainty in the inferred emitting areas from the spectral fits arises from the uncertainty in the column density $N_{\mathrm{H}}$. This conclusion is similar to the findings of Rutledge et al. (1999) for their spectral fits of the quiescent X-ray emission from accreting NS transients. This uncertainty may be reduced if high signal-to-noise spectral data becomes available in a passband that covers both the energy range where absorption is most effective and where it is not. Such observations may be possible with Chandra X-Ray Observatory and XMM-Newton.

Despite the harder tail in the spectrum produced by reprocessing of the outgoing flux through an atmosphere, spectral fits still require in most cases a hard power law component (even though its normalization is often not well constrained). In the context of the magnetar model, the origin of this power-law emission is not fully understood. Thompson \& Duncan (1996) have proposed that the non-thermal components of AXP and SGR spectra can be produced by magnetospheric currents resulting from fracturing of the neutron star crust. An important constraint on this type of model is provided by the fact that the hard emission from AXPs appears to be pulsed, often with a large amplitude. Although the angular distribution of the radiation from these effects is not yet known, a magnetospheric origin for the power-law component is appealing from the point of view of helping to account for the observed pulsations, because gravitational bending would be relatively less significant far from the stellar surface. A preliminary analysis (C. Thompson, private communication) suggests that photons produced by magnetospheric currents will preferentially escape from regions near the magnetic poles, possibly resulting in a large amplitude of pulsation. However, in order to be able to draw firmer conclusions from a timing analysis, tighter limits on the power law fraction $f_{\mathrm{pl}}$ are needed, possibly as a function of energy. To such purpose, independent constraints on the column densities would be highly desirable. In the fits in which $N_{\mathrm{H}}$ is held fixed the normalization of the power law component is much better constrained. Moreover, the column density itself influences the inferred values of the pulsed fractions (Perna, Heyl \& Hernquist 2000).

In summary, our finding that plausible atmosphere models yield thermal emitting areas consistent with a neutron star surface supports the interpretation of AXPs and SGRs as magnetars. However, based on currently available data, we cannot definitively discriminate between the magnetar and accretion models for these objects. In particular, the apparent requirement that a large fraction of the flux arises from a hard power-law component significantly complicates efforts to infer the true nature of these sources.

A variety of objections have been raised against both the magnetar and accretion models for AXPs and SGRs (e.g. Li 1999; Marsden, Rothschild \& Lingenfelter 1999; DeDeo, Psaltis \& Narayan 2000; Hulleman et al. 2000a; Marsden et al. 2001). In the case of the magnetar 
interpretation, our results show that the thermal emission is consistent with the surface area of a neutron star, provided that the spectrum from this component is sufficiently distinct from a blackbody. Moreover, the existence of a substantial non-thermal, power-law component mitigates concerns that the large pulsed fractions measured for some AXPs and SGRs might be inconsistent with the magnetar hypothesis. For an object like 1E 1048.1-5937, for example, the unusually large pulsed fraction of $\sim 70 \%$ (e.g. Corbet \& Mihara 1997; Oosterbroeck et al. 1998) may simply reflect the inferred presence of a particularly important non-thermal process whose contribution in this case approaches $\sim 80 \%$ of the total flux. It remains problematic, however, whether or not models for the origin of the power-law emission in the context of the magnetar model can account for such high relative fluxes.

Perhaps the most challenging argument against the accretion model for AXPs and SGRs comes from recent studies of optical and infrared emission from these objects. In the work of Chatterjee et al. (2000), for example, the presence of an extended disk is expected to yield significant optical and infrared fluxes (e.g. Perna et al. 2000; Perna \& Hernquist 2000), in disagreement with existing observational measurements (e.g. Hulleman et al. 2000a,b). However, Menou et al. (2001) have recently proposed an alternate accretion scenario based on fallback in which the disk always remains geometrically thin and radially compact and accretion is halted on timescales comparable to the ages of AXPs and SGRs through a thermal ionization instability. In this model, the optical and infrared emission from the disk is greatly suppressed relative to that predicted by e.g. Chatterjee et al. since the disk is limited in radial extent.

Another argument against the accretion model for AXPs concerns the unusually steady spindown of objects like RXS J1708-40 and 1E 2259+586 (Kaspi, Chakrabarty \& Steinberger 1999). Such behavior is not characteristic of accreting, binary X-ray pulsars, but the implication of this finding for isolated fallback disks is less clear, particularly for models in which the disks are low in mass (Menou et al. 2001) and considering the noisier behavior of AXP 1E 1048.1-5937 (Kaspi et al. 2000).

Thus, it appears that with existing observational constraints it is not possible to conclusively rule out either the magnetar or accretion scenario for AXPs and SGRs. In detail, however, the spectral energy distributions expected in these two classes of models should exhibit significant differences in many wavelength intervals. Hence, we are optimistic that highly sensitive ongoing multiwavelength observations combining X-ray spectroscopy with deep optical and infrared searches will eventually discriminate between the magnetar and accretion pictures, definitively revealing the true nature of these sources.

We thank Chris Thompson for enlightening discussion, Jonathan McDowell for support with the XSPEC software, and an anonymous referee for insightful comments. Support for JSH was provided by the National Aeronautics and Space Administration through Chandra Postdoctoral Fellowship Award Number PF0-10015 issued by the Chandra X-ray Observatory Center, which is operated by the Smithsonian Astrophysical Observatory for and on behalf of NASA under contract 
NAS8-39073.

\section{REFERENCES}

Alcock, C., \& Illarionov, A. 1980, ApJ, 235, 534

Alpar, M. A. 1999, astro-ph/9912228

Alpar, M. A. 2000, astro-ph/0005211

Arnaud, K. A. 1996, in ASP Conf. Series 101, Astronomical Data Analysis Software and Systems V, ed. G. Jacoby \& J. Barnes (San Francisco: ASP), 17

Brown, E. F., Bildsten, L., \& Rutledge, R. E. 1998, ApJL, 504, L195

Chatterjee, P., Hernquist, L. \& Narayan, R. 2000, ApJ, in press (CHN)

Corbet, R. \& Mihara, T. 1997, ApJ, 475, L127

Corbet, R. et al. 1995, ApJ, 443, 786

DeDeo, S., Psaltis, D. \& Narayan, R., ApJ submitted, astro-ph/0004266

Duncan, R. C. \& Thompson, C. 1992, ApJ, 392, L9

Duncan, R. C. \& Thompson, C. 1995, in AIP Conf. Proc. 366, High Velocity Neutron Stars and Gamma-Ray Bursts, ed. R. E. Rothschild \& R. E. Lingenfelter (Woodbury: AIP), 111

Ghosh, P., Angelini, L. \& White, N. E. 1997, ApJ, 478, 713

Greenstein, G., \& Hartke, G. J. 1983, ApJ, 271, 283

Heyl, J. S. \& Hernquist, L. 1997a, ApJ, 491, L95

Heyl, J. S. \& Hernquist, L. 1997b, ApJ, 489, L67

Heyl, J. S. \& Hernquist, L. 1998a, MNRAS, 298, L17

Heyl, J. S. \& Hernquist, L. 1998b, MNRAS, 300, 599

Heyl, J. S. \& Hernquist, L. 2000, MNRAS, in press

Heyl, J. S. \& Kulkarni, S. R. 1998, ApJ, 506, L61

Hulleman, F., van Kerkwijk, M. H., Verbunt, F. W., \& Kulkarni, S. R. 2000a, A\&A, 358, 60

Hulleman, F., van Kerkwijk, M. H. \& Kulkarni, S. R., Nature in press, preprint astro-ph/0011561

Hurley, K., et al. 1999, ApJ, 510, L107

Israel, G. L. et al. 1999, ApJ, 518, L110

Kaspi, V. Chakrabarty, D. \& Steinberger, J. 1999, ApJ, 525, L33

Kaspi, V.M., Gavriil, F.P., Chakrabarty, D., Lackey, J.R. \& Muno, M.P. 2000, ApJ, submitted (astro-ph/0011368)

Kouveliotou, C. et al. 1999, ApJ, 510, L115 
Li, X.-D. 1999, ApJ, 520, 271

Marsden, D., Rothschild, R. E., \& Lingenfelter, R. E. 1999, ApJ, 520, L107

Marsden, D. Lingenfelter, R. E., Rothschild, R. E. \& Higdon, J. C. 2001, ApJ in press (astro$\mathrm{ph} / 9912207)$

Menou, K., Perna, R. \& Hernquist, L. 2001, in preparation

Mereghetti, S. \& Stella, L. 1995, ApJ, 442, L17

Morrison, R., \& McCammon, D. 1983, ApJ, 270, 119

Oosterbroeck, T., Parmar, A.N., Mereghetti, S. \& Israel, G.L. 1998, A\&A, 289, 837

Pandharipande, V. 1971, Nucl. Phys., A174, 641

Pandharipande, V. \& Smith, R. A. 1971, Nucl. Phys., A237, 507

Page, D. 1995, ApJ, 442, 273

Pavlov, G. G., Shibanov, Y. A., Ventura, J., \& Zavlin, V. E. 1994, A\&A, 289, 837

Perna, R. \& Hernquist, L. 2000, ApJ, 544, L57

Perna, R., Hernquist, L. \& Narayan, R. 2000, ApJ, 541, 344

Perna, R., Heyl, J. \& Hernquist, L. 2000, ApJ, 538, L159

Potekhin, A. Y., Chabrier, G. \& Yakovlev, D. G. 1997, A\&A, 323, 415.

Rajagopal, M., \& Romani, R. W. 1996, ApJ, 461, 327

Rajagopal, M., Romani, R. W. \& Miller, M. C. 1997, ApJ, 479, 347

Romani, R. W. 1987, ApJ, 313, 718

Rutledge, R. E., Bildsten, L., Brown, E. F., Pavlov, G. G., \& Zavlin, V. E. 1999, 514, 945

Tanaka, Y., Inoue, H., \& Holt, S. S. 1994, PASJ, 46, L37

Taylor, J. H., \& Cordes, J. M. 1993, ApJ, 411, 674

Thompson, C. \& Duncan, R. C. 1996, ApJ, 473, 322

Torii, K., et al. 1998, ApJ, 503, 843

van Paradijs, J., Taam, R. E. \& van den Heuvel, E. P. J. 1995, A\&A, 299, L41

White, N. E., Angelini, L., Ebisawa, K., Tanaka, Y., \& Ghosh, P. 1996, ApJ, 463, L83

Zampieri, L., Turolla, R., Zane, S., \& Treves, A. 1995, ApJ, 439, 849

Zavlin, V. E., Pavlov, G. G., \& Shibanov, Y. A. 1996, A\& A, 315, 141 
Table 1. Log of $A S C A$ Observations Analyzed

\begin{tabular}{lcc}
\hline \hline \multicolumn{1}{c}{ Source } & \multicolumn{1}{c}{ Date } & Exposure (ks) \\
\hline \multicolumn{3}{c}{ Anomalous X-ray pulsars } \\
\hline 4U 0142+61 & 1998 August 21 & 37.7 \\
1E 1048.1-5937 & 1998 July 26 & 117.7 \\
RXS J1708-40 & 1996 September 3 & 33.7 \\
AX J1845-0258 & 1993 October 12 & 89.0 \\
& & \\
& Soft $\gamma$-ray repeaters & \\
\hline SGR 1627-41 & 1999 February 26 & 261.6 \\
SGR 1806-20 & 1993 October 10 & 108.7 \\
SGR 1900+14 & 1993 October 20 & 133.3 \\
\hline
\end{tabular}


Table 2. Spectral Fits

\begin{tabular}{|c|c|c|c|c|c|c|c|}
\hline \multirow[b]{2}{*}{ Model } & \multirow[b]{2}{*}{$N_{\mathrm{H}}\left(10^{22} \mathrm{~cm}^{-2}\right)$} & \multicolumn{2}{|c|}{ Thermal component } & \multicolumn{2}{|c|}{ Power law component } & \multirow[b]{2}{*}{$\chi_{\mathrm{red}}^{2} / N_{\mathrm{dof}}$} & \multirow[b]{2}{*}{$f_{\mathrm{pl}}^{\mathrm{b}}(\%)$} \\
\hline & & $k T(\mathrm{keV})$ & $R(\mathrm{~km})$ & $\gamma$ & $C_{1}^{\mathrm{a}}$ & & \\
\hline \multicolumn{8}{|c|}{$1 E 1048.1-5937(A X P), d=10 \mathrm{kpc}^{(\mathrm{c})}$} \\
\hline $\mathrm{BB}+\mathrm{PL}$ & $1.47_{-0.2}^{+0.14}$ & $0.63_{-0.04}^{+0.03}$ & $1.8_{-0.2}^{+0.3}$ & $3.9_{-0.3}^{+0.5}$ & $14_{-10}^{+20}$ & $0.89 / 771$ & 80 \\
\hline Magnetar+PL & $1.62_{-0.25}^{+0.34}$ & $0.43_{-0.02}^{+0.04}$ & $15.2_{-0.7}^{+4}$ & $4.9_{-0.8}^{+1.2}$ & $20_{-9}^{+23}$ & $0.89 / 771$ & 78 \\
\hline Magnetar $+\mathrm{PL}$ & 1.0 (fixed) & $0.41_{-0.01}^{+0.01}$ & $16.5_{-0.9}^{+1.3}$ & $3.4_{-0.3}^{+0.3}$ & $3.7_{-0.5}^{+0.4}$ & $0.91 / 772$ & 45 \\
\hline \multicolumn{8}{|c|}{$1 E 1845-0258(A X P), d=8.5 \mathrm{kpc}^{(\mathrm{d})}$} \\
\hline $\mathrm{BB}$ & $5.16_{-0.96}^{+1.08}$ & $0.66_{-0.06}^{+0.06}$ & $2.0_{-0.5}^{+0.8}$ & & & $0.94 / 177$ & 0 \\
\hline PL only & $10.3_{-1.5}^{+1.8}$ & & & $5.1_{-0.6}^{+0.7}$ & $974_{-828}^{+43}$ & $1.00 / 177$ & 100 \\
\hline Magnetar & $6.3_{-1.3}^{+2.1}$ & $0.41_{-0.09}^{+0.07}$ & $18_{-7.2}^{+26}$ & & & $0.97 / 177$ & 0 \\
\hline \multicolumn{8}{|c|}{$4 U 0142+61(A X P), d=1 \mathrm{kpc}^{(\mathrm{e})}$} \\
\hline $\mathrm{BB}+\mathrm{PL}$ & $1.12_{-0.08}^{+0.08}$ & $0.41_{-0.01}^{+0.02}$ & $1.7_{-0.3}^{+0.2}$ & $4.0_{-0.1}^{+0.2}$ & $333_{-60}^{+64}$ & $0.97 / 330$ & 88 \\
\hline Magnetar+PL & $0.62_{-0.14}^{+0.3}$ & $0.28_{-0.01}^{+0.01}$ & $16.1_{-0.6}^{+0.9}$ & $3.2_{-0.6}^{+0.7}$ & $44_{-31}^{+130}$ & $0.98 / 330$ & 35 \\
\hline Magnetar $+\mathrm{PL}$ & 1.0 (fixed) & $0.27_{-0.01}^{+0.01}$ & $14.4_{-1.1}^{+1.0}$ & $4.0_{-0.08}^{+0.06}$ & $216_{-10}^{+15}$ & $0.99 / 331$ & 75 \\
\hline \multicolumn{8}{|c|}{$R X S J 1708-40(A X P), d=10 \mathrm{kpc}^{(\mathrm{f})}$} \\
\hline $\mathrm{BB}+\mathrm{PL}$ & $1.47_{-0.46}^{+0.5}$ & $0.40_{-0.08}^{+0.06}$ & $11.5_{-7}^{+6}$ & $2.8_{-1}^{+0.7}$ & $36.2_{-30}^{+80}$ & $0.82 / 128$ & 73 \\
\hline Magnetar+PL & $1.24_{-0.33}^{+0.66}$ & $0.31_{-0.11}^{+0.05}$ & $69_{-33}^{+75}$ & $2.1_{-2}^{+1.4}$ & $8.2_{-8}^{+95}$ & $0.93 / 128$ & 35 \\
\hline Magnetar $+\mathrm{PL}$ & 1.0 (fixed) & $0.34_{-0.01}^{+0.02}$ & $56_{-8}^{+4}$ & $1.3_{-1.5}^{+0.8}$ & $1.7_{-1.6}^{+7}$ & $0.82 / 129$ & 25 \\
\hline \multicolumn{8}{|c|}{$S G R 1900+14, d=5 \mathrm{kpc}^{(\mathrm{g})}$} \\
\hline $\mathrm{BB}+\mathrm{PL}$ & $2.5_{-0.3}^{+0.2}$ & $0.53_{-0.16}^{+0.08}$ & $1.2_{-0.7}^{+0.8}$ & $2.1_{-0.2}^{+0.3}$ & $105_{-20}^{+80}$ & $0.83 / 309$ & 90 \\
\hline Magnetar+PL & $2.4_{-0.3}^{+0.4}$ & $0.34_{-0.16}^{+0.05}$ & $11.3_{-0.3}^{+1.4}$ & $2.1_{-0.3}^{+0.3}$ & $110_{-100}^{+70}$ & $0.83 / 309$ & 85 \\
\hline
\end{tabular}

${ }^{\text {a }}$ Power-law normalization at $1 \mathrm{keV}$ in units of $10^{-3}$ photons $\mathrm{cm}^{2} \mathrm{~s}^{-1} \mathrm{keV}^{-1}$.

${ }^{b}$ Energy flux in the power law component for the 0.7-10 keV band.

(c) Van Paradijs et al. (1995).

${ }^{(d)}$ Torii et al. (1998).

${ }^{(\mathrm{e})}$ Israel et al. (1999), but see also text.

(f) White et al. (1996).

(g) Hurley et al. (1999). 


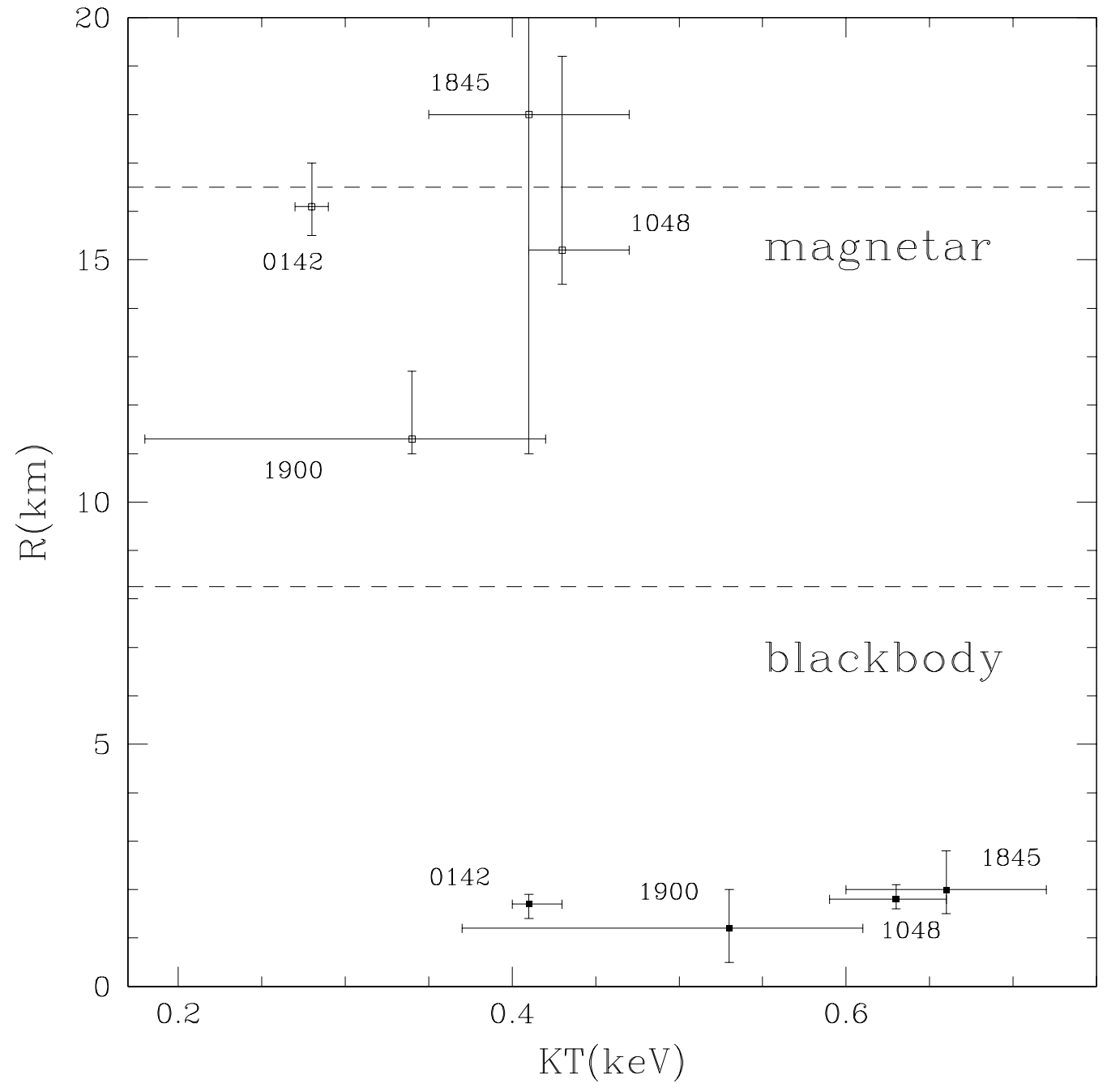

Fig. 1.- Inferred temperatures and radii for blackbody fits, and for fits done with the magnetar model. The two dashed lines mark the region of NS radii which are allowed by currently available models for the NS equation of state for a NS of $M=1.4 M_{\odot}$ (Pandharipande 1971; Pandharipande \& Smith 1975). 